\title{
Conscientiousness protects visual search performance from the impact of fatigue
}

Justin N. Grady ${ }^{1}$, Patrick H. Cox ${ }^{1,2}$, Samoni Nag ${ }^{1}$, \& Stephen R. Mitroff ${ }^{1}$

${ }^{1}$ Department of Psychological \& Brain Sciences, The George Washington University, Washington DC 20052 USA

${ }^{2}$ Intelligence Community Postdoctoral Research Fellowship Program, Department of Psychological \& Brain Sciences, The George Washington University, Washington DC 20052 USA

\section{Acknowledgements:}

This research was funded by US Army Research Office grant \#W911NF-16-1-0274) and US Army Research Laboratory Cooperative Agreements \#W911NF-19-2-0260 \& \#W911NF-21-20179. P.H.C. was supported by an appointment to the Intelligence Community Postdoctoral Research Fellowship Program at The George Washington University, administered by Oak Ridge Institute for Science and Education through an interagency agreement between the U.S. Department of Energy and the Office of the Director of National Intelligence. The authors thank Laura Schubel, Courtney Porfido, Sydni Nadler and the members of the GW Visual Cognition Lab for help with data collection, and Dwight Kravitz and Sydni Nadler for helpful discussions. Corresponding author: Stephen Mitroff, mitroff@gwu.edu

\section{Conflicts of Interest Statement:}

J.G., P.C., \& S.N. declare no conflict of interest. S.M. is a co-founder of a startup company, Kedlin Screening International, that has a product built on a platform related to the Airport Scanner app used for data collection in the current study. The company may indirectly benefit from the current findings as any other company may benefit from these publicly-available results.

\section{Data Availability Statement}

The dataset and analysis code are available on the project's OSF site (https://osf.io/8jzwc/). 


\begin{abstract}
Objective: Visual search — looking for targets among distractors - underlies many critical professions (e.g., radiology, aviation security) that demand optimal performance. As such, it is important to identify, understand, and ameliorate negative factors such as fatigue - mental and/or physical tiredness and diminished function. One way to reduce the detrimental effects is to minimize fatigue itself (e.g., scheduled breaks), but this is not always possible or sufficient.
\end{abstract}

Methods: The current study explored whether some individuals are less susceptible to the impact of fatigue than others; specifically, if conscientiousness, the ability to control impulses and plan, moderates fatigue's impact. Participants $(\mathrm{N}=374)$ completed a search task and selfreported their energy (i.e., the inverse of fatigue) and conscientiousness levels. Self-report measures were gathered as part of a large set of surveys so that participants could not anticipate any particular research question.

Results: Preregistered linear mixed effect analyses revealed main effects of energy level (lower energy reduces accuracy) and conscientiousness (more conscientiousness increases accuracy), and, critically, a significant interaction between energy level and conscientiousness. A follow-up analysis divided participants into above- vs. below-mean conscientiousness groups, revealing a significant negative relationship between energy level and accuracy for the belowmean, but not above-mean, group.

Implications: The results raise intriguing operational possibilities for visual search professions, with the most direct implication being the incorporation of conscientiousness measures to personnel selection processes

\title{
Keywords
}

Visual search, individual differences, fatigue, conscientiousness, personnel selection, energy 


\section{Introduction}

Visual search, looking for targets among distractors, is an important skill involving an array of underlying cognitive mechanisms, including perception, memory, attention, and decision making (Eckstein, 2011; Nakayama \& Martini, 2011). Search is central to many aspects of normal everyday life (e.g., finding a friend in a crowd) and is also important for a wide array of specialized tasks (e.g., soccer goalkeepers scanning the field for opposing players making runs towards the goal; Savelsbergh et al., 2002). Visual search also underlies many professional tasks that can have life-or-death outcomes, including aviation security (e.g., Mitroff et al., 2018; Wetter, 2013), medical image perception (e.g., Horowitz, 2017; Krupinski, 2015; Van der Gijp et al., 2017), lifeguarding (e.g., Lanagan-Leitzel et al., 2015), and many military operations (e.g., Nelson et al., 2015). In such professions, search often involves human operators conducting repetitive tasks in what can be mundane conditions that demand high levels of attention, vigilance, and engagement (Krupinski, 2015; Wetters, 2013). As such, operators can be susceptible to fatigue - a state of tiredness and diminished functioning.

Extensive research has explored the detrimental impact of fatigue on cognitive performance and occupational safety, suggesting that fatigue arises from an array of conditions, including long work hours, unusual shift times, and stressful employment settings, which are all common to professions involving visual search (e.g., Williamson \& Friswell, 2013). Fatigue is broadly considered to be a complex multidimensional symptom in which individuals experience physical tiredness and a lack of energy (Schwid et al, 2003). Accordingly, fatigue has an array of implications for a range of cognitive and motor tasks, with known deficits on areas such as sports performance (Smith et al., 2016), driving (Lal \& Craig, 2001), and military operations (Miller et al., 2018). 
For visual search, detriments from fatigue are generally thought to stem from a prolonged time spent on a task and/or the searchers' mental and/or physical state when starting the task (Bailey et al., 2007). While both factors are important, the current study considered participants' mental and/or physical state when they began a testing session. In professional visual search environments, each operator arrives at work in their own particular state of readiness, which can vary between and within individuals from one day to the next. An important question is how much overall operational effectiveness is affected by the operators' level of fatigue when they begin work.

Prior in-lab research has established an effect of fatigue on visual search performance. For example, sleep deprivation (one form of fatigue) has been shown to impact both accuracy and response time on visual search tasks (Santhi et al., 2007). Other studies found that fatigue impacted visual search response time, but not accuracy (De Gennaro et al., 2001; Pomplun et al., 2012). More broadly, fatigued individuals generally struggle to maintain both fast response times and high accuracy; which can lead to a speed-accuracy tradeoff wherein individuals either hold accuracy high by slowing their response time or maintain quick response times at the expense of accuracy depending on the parameters of the task. Given the emphasis of speed and precision (i.e., efficiency) in many real-world searches, it is vital that operators are both highly accurate and quick to respond.

Academic radiology and other applied research fields have consistently demonstrated an impact of fatigue on performance (e.g., Krupinski, 2015; Taylor-Phillips \& Stinton, 2019; Vosshenrich et al., 2021). For example, research has shown that radiologists' performance varies over the course of the day, generally peaking in the early evening, but suffering in the middle of the day following lunch (Monk, 2005). This "post-lunch dip effect" corresponds to a well- 
studied period of postprandial fatigue after the midday meal (Stahl et al., 1983). Similarly, research has shown that professional radiologists exhibit within-subject performance decrements following overnight shifts; diagnostic performance was worse and subjective self reports of fatigue were higher when assessed the morning after an overnight shift compared to after a normal day shift (Hanna et al., 2018). Such effects of fatigue can have profound implications for radiology, as subpar visual search performance could lead to missed abnormalities (Krupinski, 2015).

The above findings highlight a robust impact of fatigue that represents a serious concern for the successful execution of visual search in critical environments, but does this impact vary across individuals? Prior research suggests that it might; for example, there is individual variability in how vulnerable someone is to fatigue, with some groups of individuals showing more resistance to the detrimental impacts of fatigue than others (e.g., pilots compared to nonpilots; Caldwell et al., 2005). The notion that some individuals are more resilient to the negative impacts of fatigue could be leveraged to better understand the mechanisms by which fatigue affects cognition and inform operational practices such as hiring decisions.

The current study focused on one particular individual differences factor that might moderate the impact of fatigue on visual search performance: Conscientiousness - the ability to control impulses, be goal directed, plan, and delay gratification (Roberts et al., 2009).

Conscientiousness is a strong candidate trait as previous work has shown that more conscientious individuals are better searchers (Biggs et al., 2017; Spain et al., 2017). Moreover, conscientiousness may facilitate core cognitive abilities that underlie visual search performance. For example, it has been suggested that more conscientious individuals place a higher degree of emphasis on success and rule learning compared to less conscientious individuals, leading to 
higher accuracy on working memory tasks (Studer-Luethi et al., 2012) and superior performance on cognitive shifting tasks (Fleming et al., 2016). Further, previous research suggests that conscientiousness may act as a protective factor against fatigue; those with higher levels of conscientiousness were more resistant to dangerous microsleep episodes while driving after a period of sleep deprivation, compared to their less conscientious counterparts (Hidalgo-Gadea et al., 2021). As such, the goal of the current study was to explore if conscientiousness moderates the relationship between fatigue and visual search performance.

\section{Materials and Methods}

The study design and analyses were preregistered (https://osf.io/7w8dm). This research complied with the American Psychological Association Code of Ethics and was approved by the Institutional Review Board at The George Washington University (GWU). Informed consent was obtained from each participant. The data for the current study were drawn from a larger project conducted in the GWU Visual Cognition Lab wherein participants responded to self-report surveys (see Appendix) and participated in behavioral tasks. Data from this project have been used for other purposes (e.g., Nadler et al., 2021; Silverman et al., under review), and the current study only analyzed the specific subset of the data reported here.

\section{Participants}

Participants for the broader research project $(\mathrm{N}=578)$ were recruited from November 2016 to April 2021 through the GWU Department of Psychological \& Brain Sciences'subject pool and received course credit. Data collection efforts and timing were constrained by the University's semester schedule and other external influences (e.g., the COVID-19 pandemic). 
Enrolled participants could withdraw without penalty and could skip specific self-report questions. A final dataset was determined via the below ordered series of preregistered, sequential data exclusion steps.

Non-performance-based data exclusion; 578 to 559 participants

The first data exclusion step focused on non-performance-based criteria, removing data from participants who self-reported to being outside the age range of 18 to $25(\mathrm{~N}=15)$ or who completed the study twice (second datasets removed, $\mathrm{N}=4$ ).

Self-report survey performance-based data exclusion; 559 to 528 participants

The second data exclusion step removed data in three sequential steps. First, three "attention check" questions were sporadically built into the self-report surveys (see Procedures) to ensure participants read and comprehended the survey prompts. The attention checks were simple multiple-choice questions about the upcoming survey and provided feedback for wrong answers. If participants required four attempts on any one check or required seven or more (out of a possible 12) total attempts across all three checks, their full dataset was removed from all analyses $(\mathrm{N}=22)$. Second, ten of the surveys (Appendix surveys \#4-5, 9-10, 13-18) were selected, and if a participant did not respond to at least half of the questions for three or more of the surveys then their data were removed from all analyses $(\mathrm{N}=1)$. Third, for the same subset of ten surveys, participants' responses were assessed to determine if they had no variability across the entire survey. Participants' data were removed from all analyses if they clicked the same radio button for the entire survey for three or more surveys $(\mathrm{N}=8)$. 
Behavioral visual search task data exclusion; 528 to 376 participants

Of the 528 participants with usable self-report survey data, 397 also completed the behavioral visual search task. Participants' data were subsequently excluded if they failed the minimum performance-based criteria; in sequential order, data were removed if the participants did not complete exactly 72 trials of the Big Plane Challenge level (N=12), if they had a targetpresent accuracy less than $20 \%(\mathrm{~N}=6)$, or if they had a false alarm rate greater than $80 \%(\mathrm{~N}=3)$.

Survey-specific exclusion; 376 to 374 participants

A final exclusion step removed data from participants who did not have a complete dataset for the three measures of interest: energy level (i.e., the inverse of fatigue), conscientiousness, and visual search performance. Data were removed from one participant for not responding to the energy level question and one participant for not providing sufficient data for the conscientiousness measure - leaving a final dataset of 374 participants.

\section{Participant cohorts}

Participants from November 2016 to March 2020 were tested in the GWU Visual Cognition lab in person ("in-lab" cohort; N=205, Mean age=19.69 years, SD=1.19 years, 165 female, 39 male, 1 did not report gender) and participants from April 2020 to April 2021 were tested virtually using their own computer and mobile device technology ("virtual" cohort; $\mathrm{N}=169$, Mean age $=19.99$ years, $\mathrm{SD}=1.36$ years, 105 female, 62 male, 2 did not report gender). While there was no a priori reason to predict that these two cohorts would show different effects for the primary measures of interest, prior work has shown that the cohorts differ in general 
(Nadler et al., 2021). As such, cohort (in-lab vs virtual) was preregistered as a factor of no interest to be included in the planned statistical analyses.

\section{Procedure}

\section{Self-report survey data}

Participants completed a large set of self-report surveys (see Appendix) via Qualtrics, but only two were assessed for the current study. First, participants completed a "readiness survey" that asked about typical and recent sleeping habits, caffeine use, and other related factors. One question asked participants to rate their energy level (Figure 1A), with lower energy level ratings corresponding to higher fatigue and vice versa. A measure of fatigue level was calculated for visualization purposes in Figure 2 by subtracting the participants' subjective energy rating (on a 0-100 scale) from 100. Self-report measures of energy are commonly used (e.g., De Gennaro et al., 2001; LaChapelle \& Finlayson, 1998); for example, on the Stanford Sleepiness Scale (Hoddes et al., 1973) and the Visuo-analogue Sleepiness Scale (Fransson et al., 2008) participants rate how they feel within a certain range (e.g., marking how sleepy they are from "not at all" to "very much"). Prior research supports the use of such subjective reports given that individuals have insight into their own current state of fatigue - self-report measures of fatigue have been shown to directly link to objective sleepiness as measured by EEG (Åkerstedt \& Gillberg, 1990).

The second measure of interest was the conscientiousness subscale from the Big-5 Personality Inventory (John \& Srivastava, 1999). This subscale, which is calculated as the average response on a 1 to 5 scale for 9 questions, was used to measure participants' self- 
reported level of conscientiousness (Figure 1B). No other survey data, including other subscales from the Big-5 Inventory, were analyzed as part of this project as laid out in the preregistered analysis plans.

\section{Behavioral visual search task}

The visual search performance measures came from Airport Scanner (Kedlin Co.) - a mobile game wherein players search for prohibited items in simulated bags at a virtual airport security checkpoint (Figure 1C). Airport Scanner was a publicly-available game and the developers made the data available for research purposes (e.g., Ericson et al., 2017; Kramer et al., in press; Mitroff et al., 2015). Participants from the in-lab cohort completed the Airport Scanner gameplay on an iPad tablet provided to them in the testing room. The participants in the virtual cohort completed the gameplay on their own devices in their own environment.

All behavioral data for the current study came from the Big Plane Challenge in the $R \& D$ Lab level of the game; the Big Plane Challenge task was fully described in Mitroff et al. (2018). After completing two tutorial levels that provided practice and introduced the game dynamics, players completed 72 trials of gameplay. A trial was defined as a single bag that moved laterally across the screen. If participants detected a prohibited item (i.e., target), they were instructed to tap on the screen at the location of the item. If the bag did not contain any prohibited items, participants could either swipe the bag across the screen or let it move through the scanner by itself. Approximately 50\% of the trials contained a target (drawn from a set of 20 possible items) and each trial contained 5 to 15 distractor items (drawn, with replacement, from a set of 100 possible items). Participants were presented with a timer measuring total time elapsed and a score based on successful trials. Trial-level data were removed if the response time was quicker 
than $250 \mathrm{~ms}$ or longer than 10 s (21 out of 26,928 total trials removed; 17 participants had 1 outlier trial and 2 participants had 2).

\section{Primary Task Measures}

\section{A. Fatigue}

As part of a "readiness survey," participants responded to the question:

"Please rate on a scale of 0-100 your current energy level, with 0 meaning you have no energy and can fall asleep at any moment and 100 meaning you feel your best. Using this scale, how do you feel right now?"

High energy rating $=$ low fatigue

\section{B. Conscientiousness}

For the Big-5 Personality Inventory (John \& Srivastava, 1999), participants used a 5-point scale (from strongly agree to strongly disagree) to rate 44 statements for how much each represents them. Conscientiousness was calculated as the average responses to these 9:

$\begin{array}{ll}\text { Does a thorough job } & \text { Perseveres until the task is finished } \\ \text { Can be somewhat careless* } & \text { Does things efficiently } \\ \text { Is a reliable worker } & \text { Makes plans and follows through with them } \\ \text { Tends to be disorganized* } & \text { Is easily distracted* } \\ \text { Tends to be lazy* } & \end{array}$

Score range of 1 to $5 ; 1=$ low conscientiousness; statements with an * are reverse coded

\section{Visual Search}

Sample target-present trials from Airport Scanner's Big Plane Challenge (Kedlin Co.; images used with permission). targets are Large Water bottle, knife, and dynamite stick, respectively.
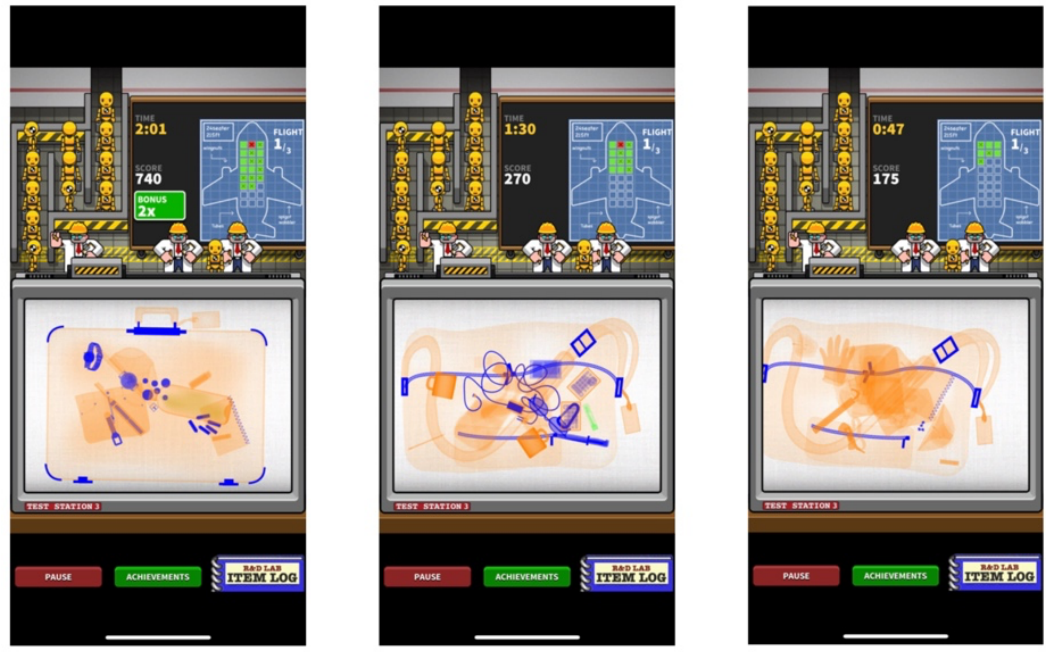

Dependent variables of interest were target-present accuracy and response time

Figure 1. Study measures of (A) energy (i.e., the inverse of fatigue), (B) conscientiousness, and (C) visual search. 


\section{Planned analyses}

As preregistered, linear mixed effect (LME) models were used to assess target-present accuracy and target-present response time as dependent variables with energy level, conscientiousness, and their interaction as the fixed effects of interest. Gender (male, female, no response) and participant cohort (in-lab, virtual) were assessed as categorical random effects of no interest. The LME models were defined as: accuracy [or response time] $\sim 1+$ energy level + conscientiousness + energy level ${ }^{*}$ conscientiousness $+(1 /$ gender $)+(1 /$ cohort $)$. The interaction effect of energy level and conscientiousness for each model was of primary interest, given the hypothesis that the effect of fatigue on visual search performance would be moderated by conscientiousness.

\section{Results}

\section{Descriptive results}

The average energy level rating was $62.56(\mathrm{SD}=19.29$, range $=10$ to 100$)$, and the average conscientiousness score was 3.55 ( $\mathrm{SD}=0.58$, range=2.22 to 5). The average visual search targetpresent accuracy was $71.56 \%(\mathrm{SD}=14.13 \%$, range $=21.05 \%$ to $97.37 \%)$, and the average visual search target-present response time was $2713.56 \mathrm{~ms}(\mathrm{SD}=502.99 \mathrm{~ms}$, range $=1574.74 \mathrm{~ms}$ to $4459.63 \mathrm{~ms})$.

\section{Linear mixed effect (LME) models}

The LME model for target-present accuracy produced a significant main effect of the energy level $(F(1,370)=5.071 ; p=0.0249)$ such that higher levels of fatigue related to lower 
accuracy, a main effect of conscientiousness $(F(1,370)=4.516 ; p=0.034)$ such that higher levels of conscientiousness related to higher accuracy, and a significant interaction $(F(1,370)=4.117$; $p=0.043)$. The LME model for target-present response time produced no significant main effects (energy level: $F(1,370)=2.312 ; p=0.129$; conscientiousness: $F(1,370)=2.486 ; p=0.116)$, nor a significant interaction $(F(1,370)=1.993 ; p=0.159)$.

\section{Follow-up analyses}

Given the significant interaction between energy level and conscientiousness in the LME model of accuracy, follow up analyses were conducted to delineate the nature of this relationship. The participants were divided into above-mean and below-mean conscientiousness groups based on a mean split of the conscientiousness scores, and separate correlation analyses of visual search accuracy as a function of fatigue were conducted for each group. The split was done with the mean, instead of the median, since 22 participants fell at the median value for conscientiousness. Though the primary purpose of this analysis was to illustrate the nature of the significant interaction effect (Figure 2), the correlations for each group were also compared using Fisher's $r$ to $z$ transformation. The above-mean conscientiousness group had an average conscientiousness score of $4.08(\mathrm{SD}=0.34)$, and there was not a significant correlation between fatigue and accuracy $(\mathrm{N}=191, r=0.034, p=0.644)$. The below-mean conscientiousness group had an average conscientiousness score of $3.05(\mathrm{SD}=0.33)$, and there was a significant correlation between fatigue and accuracy $(\mathrm{N}=183, r=-0.176, p=0.017)$, with higher fatigue corresponding to lower visual search accuracy. The two correlations significantly differed from one another; $\mathrm{z}=2.03, p=0.042$. 


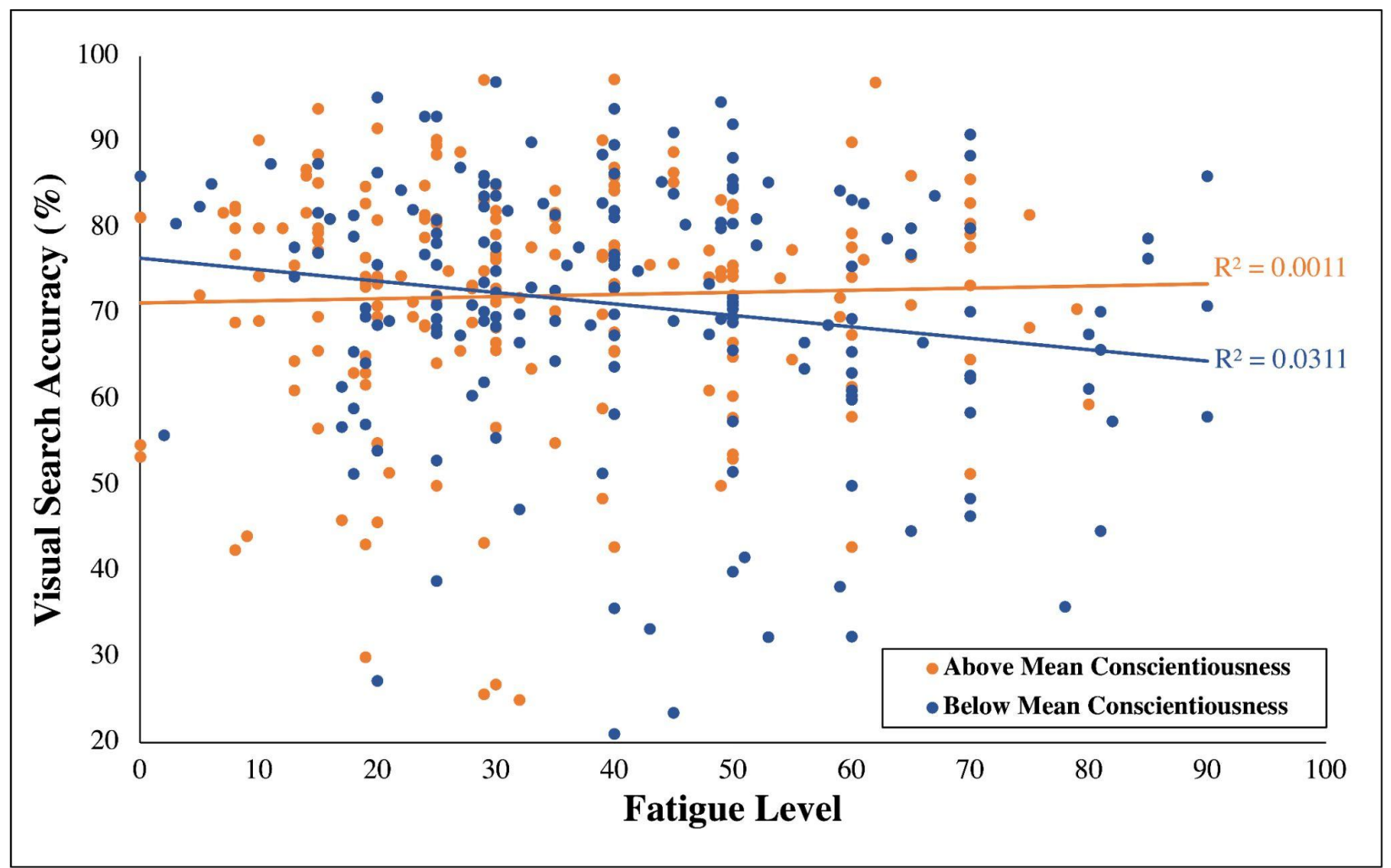

Figure 2. Target-present accuracy by fatigue (the inverse of the measured energy level), separated for the participants with above-mean (orange) and below-mean (blue) conscientiousness scores. The vertical axis range begins at 20 given that data exclusion parameters removed visual search data from participants with an average performance below $20 \%$.

\section{Discussion}

The current study used an individual differences approach to explore the relationship between fatigue and visual search performance. Fatigue negatively affects search (Bailey et al., 2007; De Gennaro et al., 2001; Santhi et al., 2007), which has profound implications for a wide swath of professions, including aviation security, radiology, lifeguarding, and more. Given that a mistake in such professions could have life-or-death consequences, it is important to know which factors may help to combat fatigue-induced performance decrements.

This study first contributed to the extant literature by providing supporting data that fatigue negatively related to visual search accuracy. Specifically, higher levels of fatigue 
corresponded to lower accuracy, which is in line with previous results (Hanna et al., 2018; Santhi et al., 2007). Second, there was also a significant main effect of conscientiousness (higher levels of conscientiousness related to higher visual search accuracy), which supports prior results (Biggs et al., 2017; Spain et al., 2017). Finally, the novel finding of the current study was that the impact of fatigue on search accuracy was moderated by conscientiousness. It was found that individuals who self-reported higher levels of conscientiousness did not demonstrate a significant effect of fatigue on accuracy while those who self-reported lower levels of conscientiousness did have a significant relationship between fatigue and accuracy, with higher fatigue relating to lower accuracy (Figure 2).

There was no observed significant relationship between fatigue and response time, yet previous work has found such a link (De Gennaro et al., 2001). There are several possible explanations for this, including differences in the task design. For example, a speed-accuracy trade-off, where accuracy is held high at the expense of response time, is more commonly found in self-paced tasks when participants have time to initiate a response (Wilkinson, 1969). The dynamics of the visual search task employed here contained both self-paced and experimenterpaced aspects, making room for multiple possible outcomes. Likewise, it could be that the current task was not demanding enough to require an increase in response time; it may be that higher conscientious participants were able to increase cognitive effort without sacrificing response time to hold their accuracy high.

\section{Implications for cognitive psychology literature}

Individual differences have become an area of great interest for cognitive psychology research, and this study offers an example of gaining insight by accounting for a personality 
difference. It is worth noting that while conscientiousness was the sole individual differences measure assessed here, it is possible that other measures could also play a meaningful role. Likewise, the current study was cross-sectional, and it could be informative to implement a longitudinal study that assesses the same individuals multiple times. This is especially intriguing given that the primary result from the current study was that a trait-like factor (conscientiousness) impacted a state-like factor (fatigue); there might be mechanistically informative nuances revealed by exploring other trait-state relationships.

More broadly, it is worth considering the nature of the currently employed study procedures and how they might offer a roadmap for future academic research efforts. This study took advantage of an extensive data collection effort that tested a large cohort of participants on a wide range of assessments (Appendix). One key advantage of this process is that there was no indication to the participants of any particular experimental goal or hypothesis. In fact, the current experimental hypothesis was developed after the data collection effort. A potential downside of this process, however, is that a research team could assess multiple hypotheses and then choose to only publish a subset; which could result in false discoveries. However, the critical step of preregistration alleviates this concern. The current study, for example, preregistered analyses and only assessed the subset of data a priori identified as of interest. This experimental procedure thus allows for gathering unbiased data that addresses a number of potential variables of interest, and then later preregistering specific research questions to explore. This process may prove quite useful, especially for individual differences research questions. 


\section{Implications for visual search professions}

The current findings have the potential to inform professional operations that rely on visual search. For example, knowing that more conscientious individuals are less susceptible to the negative impacts of fatigue could inform hiring and staffing decisions for aviation security, military operations, lifeguarding, and more. A fair question, though, is just how operationally meaningful is this particular effect; is the impact of the fatigue-conscientiousness relationship relevant for visual search professions, and should they consider incorporating this knowledge into their operational plans? Prior work using the same experimental paradigm provides insight to this issue. Specifically, in a prior study (Mitroff et al., 2018), US airport screening officers completed a visual search task that was nearly identical to the one used in the current study. Critically, the officers' performance in that task significantly correlated with their on-job performance such that those who were better at the visual search task were both more accurate and quicker at actual checkpoints. This suggests that this paradigm is sensitive to operational outcomes, and the current results demonstrate that this paradigm is also sensitive to the impacts of fatigue and conscientiousness. As such, it is reasonable to suggest that these data offer a potentially generalizable outcome with practical implications.

\section{Conclusions}

The current project found that fatigue negatively impacted search performance, but that this relationship was significantly moderated by conscientiousness such that more conscientious individuals showed no relationship between fatigue and search accuracy. This exciting, but 
straightforward, result could have clear and direct implications for many professional settings that rely on visual search. 


\section{Key Points}

- Higher levels of self-reported fatigue relates to lower visual search accuracy

- Higher self-reported conscientiousness relates to higher visual search accuracy

- Fatigue and conscientiousness interact such that more conscientious individuals do not show a relationship between fatigue and search accuracy

\section{References}

Åkerstedt, T., \& Gillberg, M. (1990). Subjective and objective sleepiness in the active individual. International Journal of Neuroscience, 52(1-2), 29-37.

Bailey, A., Channon, S., \& Beaumont, J. G. (2007). The relationship between subjective fatigue and cognitive fatigue in advanced multiple sclerosis. Multiple Sclerosis Journal, 13(1), 7380.

Biggs, A. T., Clark, K., \& Mitroff, S. R. (2017). Who should be searching? Differences in personality can affect visual search accuracy. Personality and Individual Differences, 116, 353-358.

Caldwell, J. A., Mu, Q., Smith, J. K., Mishory, A., Caldwell, J. L., Peters, G., ... \& George, M. S. (2005). Are individual differences in fatigue vulnerability related to baseline differences in cortical activation?. Behavioral Neuroscience, 119(3), 694.

De Gennaro, L., Ferrara, M., Curcio, G., \& Bertini, M. (2001). Visual search performance across $40 \mathrm{~h}$ of continuous wakefulness: Measures of speed and accuracy and relation with oculomotor performance. Physiology \& Behavior, 74(1-2), 197-204.

Eckstein, M. P. (2011). Visual search: A retrospective. Journal of Vision, 11(5), 14-14. 
Ericson, J. M., Kravitz, D. J., \& Mitroff, S. R. (2017). Visual search: You are who you are (+ a learning curve). Perception, 46(12), 1434-1441.

Fleming, K. A., Heintzelman, S. J., \& Bartholow, B. D. (2016). Specifying associations between conscientiousness and executive functioning: Mental set shifting, not prepotent response inhibition or working memory updating. Journal of Personality, 84(3), 348-360.

Fransson, P. A., Patel, M., Magnusson, M., Berg, S., Almbladh, P., \& Gomez, S. (2008). Effects of 24-hour and 36-hour sleep deprivation on smooth pursuit and saccadic eye movements. Journal of Vestibular Research, 18(4), 209-222.

Hanna, T. N., Zygmont, M. E., Peterson, R., Theriot, D., Shekhani, H., Johnson, J. O., \& Krupinski, E. A. (2018). The effects of fatigue from overnight shifts on radiology search patterns and diagnostic performance. Journal of the American College of Radiology, 15(12), 1709-1716.

Hidalgo-Gadea, G., Kreuder, A., Krajewski, J., \& Vorstius, C. (2021). Towards better microsleep predictions in fatigued drivers: exploring benefits of personality traits and IQ. Ergonomics, 64(6), 778-792.

Hoddes, E., Zarcone, V., Smythe, H., Phillips, R., \& Dement, W. C. (1973). Quantification of sleepiness: a new approach. Psychophysiology, 10(4), 431-436.

Horowitz, T. S. (2017). Prevalence in visual search: From the clinic to the lab and back again. Japanese Psychological Research, 59(2), 65-108.

John, O. P., \& Srivastava, S. (1999). The Big-Five trait taxonomy: History, measurement, and theoretical perspectives (Vol. 2, pp. 102-138). Berkeley: University of California. 
Kramer, M. R., Cox, P. H., Mitroff, S. R., \& Kravitz, D. J. (in press). A precise quantification of how prior experience informs current behavior. Journal of Experimental Psychology: General.

Krupinski, E. A. (2015). Improving patient care through medical image perception research. Policy Insights from the Behavioral and Brain Sciences, 2(1), 74-80.

LaChapelle, D. L., \& Finlayson, M. A. J. (1998). An evaluation of subjective and objective measures of fatigue in patients with brain injury and healthy controls. Brain injury, 12(8), 649-659.

Lal, S. K., \& Craig, A. (2001). A critical review of the psychophysiology of driver fatigue. Biological Psychology, 55(3), 173-194.

Lanagan-Leitzel, L. K., Skow, E., \& Moore, C. M. (2015). Great expectations: Perceptual challenges of visual surveillance in lifeguarding. Applied Cognitive Psychology, 29(3), 425435.

Miller, N. L., Matsangas, P., \& Shattuck, L. G. (2018). Fatigue and its effect on performance in military environments. In Performance Under Stress (pp. 247-266). CRC Press.

Mitroff, S. R., Biggs, A. T., Adamo, S. H., Dowd, E. W., Winkle, J., \& Clark, K. (2015). What can 1 billion trials tell us about visual search? Journal of Experimental Psychology: Human Perception \& Performance, 41(1), 1-5. DOI: 10.1037/xhp0000012

Mitroff, S. R., Ericson, J. M., \& Sharpe, B. (2018). Predicting airport screening officers' visual search competency with a rapid assessment. Human Factors, 60(2), 201-211.

Monk, T. H. (2005). The post-lunch dip in performance. Clinics in Sports Medicine, 24(2), e15e23. 
Nadler, S. M., Nag, S., \& Mitroff, S. R. (2021, November). What to do with pandemic participant data? Differences in behavioral, compliance, and self-report survey data between pre- and peri-pandemic participant cohorts. Poster presented at the annual Object Perception, Attention, and Memory meeting.

Nakayama, K., \& Martini, P. (2011). Situating visual search. Vision Research, 51(13), 15261537.

Nelson, J. M., McKinley, R. A., McIntire, L. K., Goodyear, C., \& Walters, C. (2015). Augmenting visual search performance with transcranial direct current stimulation (tDCS). Military Psychology, 27(6), 335-347.

Pomplun, M., Silva, E. J., Ronda, J. M., Cain, S. W., Münch, M. Y., Czeisler, C. A., \& Duffy, J. F. (2012). The effects of circadian phase, time awake, and imposed sleep restriction on performing complex visual tasks: Evidence from comparative visual search. Journal of Vision, 12(7), 14-14.

Roberts, B. W., Jackson, J. J., Fayard, J. V., Edmonds, G., \& Meints, J. (2009).

Conscientiousness. In M. R. Leary \& R. H. Hoyle (Eds.), Handbook of Individual Differences in Social Behavior (pp. 369-381). The Guilford Press.

Santhi, N., Horowitz, T. S., Duffy, J. F., \& Czeisler, C. A. (2007). Acute sleep deprivation and circadian misalignment associated with transition onto the first night of work impairs visual selective attention. PloS ONE, 2(11), e1233.

Savelsbergh, G. J., Williams, A. M., Kamp, J. V. D., \& Ward, P. (2002). Visual search, anticipation and expertise in soccer goalkeepers. Journal of Sports Sciences, 20(3), 279-287. 
Schwid, S. R., Tyler, C. M., Scheid, E. A., Weinstein, A., Goodman, A. D., \& McDermott, M. P. (2003). Cognitive fatigue during a test requiring sustained attention: a pilot study. Multiple Sclerosis Journal, 9(5), 503-508.

Silverman, M. S., Nag, S., Kalishman, A., Cox, P. H., \& Mitroff, S. R., (under review). Symptoms associated with obsessive-compulsive disorder among university students during the covid-19 pandemic. Personality and Individual Differences.

Smith, M. R., Zeuwts, L., Lenoir, M., Hens, N., De Jong, L. M., \& Coutts, A. J. (2016). Mental fatigue impairs soccer-specific decision-making skill. Journal of Sports Sciences, 34(14), 1297-1304.

Spain, R. D., Hedge, J. W., \& Blanchard, J. K. (2017, September). Examining predictors of visual search success in transportation security officers and behavior detection officers. In Proceedings of the Human Factors and Ergonomics Society Annual Meeting (Vol. 61, No. 1, pp. 1308-1312). Sage CA: Los Angeles, CA: SAGE Publications.

Stahl, M. L., Orr, W. C., \& Bollinger, C. (1983). Postprandial sleepiness: objective documentation via polysomnography. Sleep, 6(1), 29-35.

Studer-Luethi, B., Jaeggi, S. M., Buschkuehl, M., \& Perrig, W. J. (2012). Influence of neuroticism and conscientiousness on working memory training outcome. Personality and Individual Differences, 53(1), 44-49.

Taylor-Phillips, S., \& Stinton, C. (2019). Fatigue in radiology: a fertile area for future research. The British Journal of Radiology, 92(1099), 20190043.

Van der Gijp, A., Ravesloot, C. J., Jarodzka, H., Van der Schaaf, M. F., Van der Schaaf, I. C., van Schaik, J. P., \& Ten Cate, T. J. (2017). How visual search relates to visual diagnostic 
performance: a narrative systematic review of eye-tracking research in radiology. Advances in Health Sciences Education, 22(3), 765-787.

Vosshenrich, J., Brantner, P., Cyriac, J., Boll, D. T., Merkle, E. M., \& Heye, T. (2021). Quantifying Radiology Resident Fatigue: Analysis of Preliminary Reports. Radiology, 298(3), 632-639.

Wetter, O. E. (2013). Imaging in airport security: Past, present, future, and the link to forensic and clinical radiology. Journal of Forensic Radiology and Imaging, 1(4), 152-160.

Wilkinson, R. (1969). Some factors influencing the effect of environmental stressors upon performance. Psychological Bulletin, 72(4), 260.

Williamson, A., \& Friswell, R. (2013). Fatigue in the workplace: causes and countermeasures. Fatigue: Biomedicine, Health \& Behavior, 1(1-2), 81-98. 


\section{Appendix}

The current study drew data from a broader research project that involved administering a large set of self-report surveys and behavioral measures.

Self-report surveys

Participants completed the below self-report surveys as part of their participation in the project, but the only data analyzed in the current study were one question from the "readiness survey" (\#2) and the conscientiousness measure from the Personality Inventory (\#17).

1. Basic Demographics Survey (12 questions)

Survey constructed in GW Visual Cognition lab

2. Readiness Survey (14 questions)

Questions about sleep, caffeine use, energy, and related influences on readiness. Survey constructed in GW Visual Cognition lab

3. Modified Edinburgh Handedness Inventory (13 questions)

Edlin, J. M., Leppanen, M. L., Fain, R. J., Hackländer, R. P., Hanaver-Torrez, S. D., \&

Lyle, K. B. (2015). On the use (and misuse?) of the Edinburgh Handedness Inventory. Brain and Cognition, 94, 44-51.

4. State-Trait Anxiety Inventory (40 questions)

Spielberger, C. D., Gorsuch, R. L., Lushene, R., Vagg, P. R., \& Jacobs, G. A. (1983). Manual for the State-Trait Anxiety Inventory. Palo Alto, CA: Consulting Psychologists Press.

5. Adult ADD/ADHD Questionnaire (24 questions) 
Jasper, L., \& Goldberg, I. (1993). Jasper/Goldberg Adult ADD Questionnaire Retrieved October 1, 2009, from http://www.mentalhelp.net/poc/view_doc.php?id= 974\&type $=$ doc $\&$ cn $=$ ADHD

6. Concussion history (29 questions)

Survey constructed in GW Visual Cognition lab

7. Hyperfocusing/flow survey (19 questions)

Csikszentmihalyi, Mihaly (Ed); Csikszentmihalyi, Isabella Selega (Ed). (1988). Optimal experience: Psychological studies of flow in consciousness (pp. 15-35). New York, NY, US: Cambridge University Press, xiv, 416 pp.

8. Video-game questionnaire (77 questions)

Green, C. S., Kattner, F., Eichenbaum, A., Bediou, B., Adams, D. M., Mayer, R. E., \& Bavelier, D. (2017). Playing some video games but not others is related to cognitive abilities: A critique of Unsworth et al. (2015). Psychological Science, 28(5), 679-682. https://journals.sagepub.com/doi/suppl/10.1177/0956797616644837/suppl_file/VideoGame-Expertise_Classification_Scheme.pdf

9. Maximization Scale (13 questions)

Schwartz, B., Ward, A., Monterosso, J., Lyubomirsky, S., White, K., \& Lehman, D. R. (2002). Maximizing versus satisficing: happiness is a matter of choice. Journal of Personality and Social Psychology, 83(5), 1178.

10. Barratt Impulsivity Scale (30 questions)

Patton, J. H., Stanford, M. S., \& Barratt, E. S. (1995). Factor structure of the Barratt impulsiveness scale. [Research support, non-U.S. Gov't]. Journal of Clinical Psychology, $51(6), 768-774$. 
11. Pittsburgh Sleep Quality Index (20 questions)

Buysse, D. J., Reynolds III, C. F., Monk, T. H., Berman, S. R., \& Kupfer, D. J. (1989). The Pittsburgh Sleep Quality Index: A new instrument for psychiatric practice and research. Psychiatry Research, 28(2), 193-213.

12. Pastimes (18 questions)

Survey constructed in GW Visual Cognition lab

13. Autism-spectrum Quotient (50 questions)

Baron-Cohen, S., Wheelwright, S., Skinner, R., Martin, J., \& Clubley, E. (2001). The autism-spectrum quotient (AQ): Evidence from asperger syndrome/high-functioning autism, males and females, scientists and mathematicians. Journal of Autism and Developmental Disorders, 31(1), 5-17.

14. Responsibility Attitudes Scale (26 questions)

Salkovskis, P. M., Wroe, A. L., Gledhill, A., Morrison, N., Forrester, E., Richards, C., ... \& Thorpe, S. (2000). Responsibility attitudes and interpretations are characteristic of obsessive compulsive disorder. Behaviour Research and Therapy, 38(4), 347-372.

15. Obsessive-Compulsive Scale (20 questions)

Abramowitz, J. S., Deacon, B. J., Olatunji, B. O., Wheaton, M. G., Berman, N. C., Losardo, D., ... \& Hale, L. R. (2010). Assessment of obsessive-compulsive symptom dimensions: development and evaluation of the Dimensional Obsessive-Compulsive Scale. Psychological Assessment, 22(1), 180.

16. Grit (10 questions) 
Duckworth, A. L., Peterson, C., Matthews, M. D., \& Kelly, D. R. (2007). Grit:

perseverance and passion for long-term goals. Journal of Personality and Social Psychology, 92(6), 1087.

17. Personality Inventory - Big Five Inventory (44 questions)

John, O. P., \& Srivastava, S. (1999). The Big-Five trait taxonomy: History, measurement, and theoretical perspectives (Vol. 2, pp. 102-138). Berkeley: University of California.

18. Self-control Scale (35 questions)

Tangney, J. P., Baumeister, R. F., \& Boone, A. L. (2004). High self-control predicts good adjustment, less pathology, better grades, and interpersonal success. Journal of Personality, 72(2), 271-324.

19. Media Multitasking Measure-Short (9 questions)

Baumgartner, S. E., Lemmens, J. S., Weeda, W. D., \& Huizinga, M. (2017). Measuring media multitasking. Development of a short measure of media multitasking. Journal of Media Psychology, 29(2), 92-101.

\section{Behavioral tasks}

Participants from the in-lab and virtual cohorts completed the Airport Scanner task described in the main text. Participants from the in-lab cohort also completed additional behavior tasks that were not analyzed in the current study:

1. Simple reaction time task

Participants made a speeded response to a visual stimulus to obtain a baseline measure of reaction timing. 
2. Continuous performance task (AX-CPT)

Task similar to: Cohen, J.D., Barch, D.M., Carter, C.S., \& Servan-Schreiber, D. (1999). Schizophrenic deficits in the processing of context: Converging evidence from three theoretically motivated cognitive tasks. Journal of Abnormal Psychology, 108, 120-133.

3. Visual working memory task

Task similar to: Luck, S. J., \& Vogel, E. K. (1997). The capacity of visual working memory for features and conjunctions. Nature, 390(6657), 279-281. 